\title{
IRREGULAR DIFFERENTIAL SYSTEMS OF ORDER TWO AND THE RELATED EXPANSION PROBLEMS*
}

\author{
BY
}

M. H. STONE

We have prevously discussed the similarities between the series of Fourier and of Birkhoff. $\dagger$ Since a series of Birkhoff is defined by a linear homogeneous differential system of the $n$th order in which the boundary conditions are of regular type, $\ddagger$ it is natural to attempt an extension of the methods there employed to some systems with irregular boundary conditions. We shall discuss here the case $n=2$, with the hope of giving a comparatively exhaustive treatment of the narrowed topic. From our point of view, it is not essential in this discussion that a series be treated with regard to its convergence: a sum by appropriate means we consider equally valuable. A treatment of the convergence of the formal expansions for a function restricted to have a certain number of derivatives and to satisfy certain boundary conditions has come to our attention since the completion of this paper.§ As Professor Jackson has suggested to the writer, the methods of Wilder in a similar problem could be applied to this end, as is obvious from a comparison of the formulas of this paper with his.|l It should be noted, however, that under our discussion of systems of type 1, Case I, the series for the function 1 can be seen to be divergent, so that such results are not so useful as it might appear. We note that the series discussed in this paper are entirely different from those discussed by Jackson and Hopkins in the case $n \geqq 3 . \Uparrow$

* Presented to the Society, December 30, 1924; accepted in partial fulfillment of the requirements for the degree of Doctor of Philosophy at Harvard University; received by the editors of these Transactions in October, 1925.

† Stone, these Transactions, vol. 28 (1926), pp. 695-761.

$\ddagger$ Birkhoff, these Transactions, vol. 9 (1908), p. 383.

\& Pollaczek-Geiringer, Mathematische Annalen, vol. 90 (1923), pp. 292-317.

|| C. E. Wilder, these Transactions, vol. 18 (1917), pp. 415-442.

If Hopkins, these Transactions, vol. 20 (1919), pp. 245-259; Jackson, Proceedings of the American Academy of Arts and Sciences, vol. 51 (1915-1916), pp. 383-417. 


\section{Classification of the boundary conditions}

Our first task is clearly that of separating all possible boundary conditions, $n=2$, into regular and irregular types. We shall refer to Birkhoff's memoir, cited above, for the definition of regularity, and for the fact that in the case where $n$ is even the several conditions of that definition reduce to one. We then prove

THEOREM I. The only irregular boundary conditions for $n=2$ are of the form

$$
\begin{aligned}
u^{\prime}(0)+A u^{\prime}(1)+B u(1) & =0 \\
u(0)-A u(1) & =0
\end{aligned}
$$

or of the form

$$
\begin{aligned}
A u^{\prime}(0)-u^{\prime}(1)+B u(0) & =0, \\
A u(0)+u(1) & =0,
\end{aligned}
$$

where $A, B$ are real or complex constants.* The two differential syslems obtained by adjoining to the equation

$$
u^{\prime \prime}+(\lambda+g) u=0, \quad g(x) \text { summable }, \quad 0 \leqq x \leqq 1,
$$

the boundary conditions (1) and (2) respectively, are adjoint systems.

The general boundary conditions, $n=2$, can be written

$$
\begin{aligned}
& a_{1} u^{\prime}(0)+b_{1} u^{\prime}(1)+c_{1} u(0)+d_{1} u(1)=0, \\
& a_{2} u^{\prime}(0)+b_{2} u^{\prime}(1)+c_{2} u(0)+d_{2} u(1)=0,
\end{aligned}
$$

where $a_{1}, b_{1}, c_{1}, d_{1}, a_{2}, b_{2}, c_{2}, d_{2}$ are any real or complex constants such that the linear forms of which they are the coefficients remain linearly independent. We must consider several cases.

Case I. $a_{1} b_{2}-a_{2} b_{1} \neq 0$. Employing the notation of Birkhoff's definition of regularity, we write

$$
\theta_{0}+\theta_{1} s+\frac{\theta_{2}}{s} \equiv\left|\begin{array}{cc}
\left(a_{1}+b_{1} s\right) i & -\left(a_{1}+\frac{b_{1}}{s}\right) i \\
\left(a_{2}+b_{2} s\right) i & -\left(a_{2}+\frac{b_{2}}{s}\right) i
\end{array}\right| \equiv\left(\frac{1}{s}-s\right)\left(a_{1} b_{2}-a_{2} b_{1}\right)
$$

Since $\theta_{1} \theta_{2} \neq 0$, the boundary conditions are regular.

- Tamarkin, Rendiconti del Circolo Matematico di Palermo, vol. 34 (1912), pp. 360-361. 
Case II. $a_{1} b_{2}-a_{2} b_{1}=0 ; a_{1}$ and $b_{1}$ not both zero. The boundary conditions can be reduced by linear combination to the form

Then

$$
\begin{aligned}
a_{1} u^{\prime}(0)+b_{1} u^{\prime}(1)+c_{1} u(0)+d_{1} u(1) & =0, \\
c_{2} u(0)+d_{2} u(1) & =0 .
\end{aligned}
$$

$$
\begin{aligned}
& \theta_{0}+\theta_{1} s+\theta_{2} / s \equiv\left|\begin{array}{rr}
i\left(a_{1}+b_{1} s\right) & -i\left(a_{1}+b_{1} / s\right) \\
\left(c_{2}+d_{2} s\right) & \left(c_{2}+d_{2} / s\right)
\end{array}\right| \equiv 2\left(a_{1} c_{2}+b_{1} d_{2}\right) \\
& +\left(a_{1} d_{2}+b_{1} c_{2}\right)(1 / s+s) \text {. }
\end{aligned}
$$

If $a_{1} d_{2}+b_{1} c_{2} \neq 0$, the conditions are regular; on the other hand, if $a_{1} d_{2}+b_{1} c_{2}=0$, the conditions are irregular. In this latter case, since $c_{2}$ and $d_{2}$ cannot both vanish, we have left

$$
\begin{aligned}
a_{1} u^{\prime}(0)+b_{1} u^{\prime}(1)+c_{1} u(0)+d_{1} u(1) & =0, \\
a_{1} u(0)-b_{1} u(1) & =0 .
\end{aligned}
$$

If these boundary conditions have $a_{1} \neq 0$ they can be reduced to the form (1); if $b_{1} \neq 0$, to the form (2).

Case III. $a_{1}=a_{2}=b_{1}=b_{2}=0$. Since the boundary conditions

$$
\begin{aligned}
& c_{1} u(0)+d_{1} u(1)=0, \\
& c_{2} u(0)+a_{2} u(1)=0
\end{aligned}
$$

are linearly independent they reduce to $u(0)=u(1)=0$, a well known regular case.

It is now a matter of simple computation to show that the two differential systems defined in the latter part of the theorem are actually adjoint. The method employed is sufficiently familiar that we omit details.*

\section{ThE IRREgULAR BOUNDARY VALUE PROBLEM}

We are now prepared to consider the boundary value problem in the irregular cases of Theorem $I$; that is, to investigate the values of $\lambda$ for which the differential systems have solutions not identically zero. As is well known, these characteristic values are the same for a system and its adjoint. On setting $\lambda=\rho^{2}$, the two systems are

$$
\begin{aligned}
u^{\prime \prime}+\left(\rho^{2}+g\right) u & =0, & u^{\prime \prime}+\left(\rho^{2}+g\right) u & =0, \\
u^{\prime}(0)+A u^{\prime}(1)+B u(1) & =0, & A u^{\prime}(0)-u^{\prime}(1)+B u(0) & =0, \\
u(0)-A u(1) & =0, & A u(0)+u(1) & =0 .
\end{aligned}
$$

* Bócher, Leçons sur les Methodes de Sturm, Paris, 1917, Chapter II. 
The characteristic values in $\rho$ may be found as the roots of the equation

$$
\left|\begin{array}{ll}
u_{1}^{\prime}(0)+A u_{1}^{\prime}(1)+B u_{1}(1) & u_{2}^{\prime}(0)+A u_{2}^{\prime}(1)+B u_{2}(1) \\
u_{1}(0)-A u_{1}(1) & u_{2}(0)-A u_{2}(1)
\end{array}\right|=0
$$

where $u_{1}, u_{2}$ are any two linearly independent solutions of the differential equation defined for all values of $\rho$. The existence of such solutions has been demonstrated in our preceding paper.

A number of outstanding facts are revealed at once if we choose $u_{1}, u_{2}$ as solutions satisfying the boundary conditions

$$
u_{1}(0)=1, u_{1}^{\prime}(0)=0, u_{2}(0)=0, u_{2}^{\prime}(0)=1 \text {. }
$$

On expanding the determinant and making use of the fact that

$$
u_{1}(1) u_{2}^{\prime}(1)-u_{1}^{\prime}(1) u_{2}(1)=u_{1}(0) u_{2}^{\prime}(0)-u_{1}^{\prime}(0) u_{2}(0)=1,
$$

we find the equation

$$
\left(A^{2}-1\right)+A\left(u_{1}(1)-u_{2}^{\prime}(1)\right)-B u_{2}(1)=0 .
$$

If $A=B=0$, there can be no characteristic values; henceforth this possibility shall be excluded. Again, if $A^{2}-1=0, B=0$, the equation is $u_{1}(1)-u_{2}^{\prime}(1)=0$, whence we conclude that a root in this case cannot be a root in the case $A^{2}-1 \neq 0, B=0$. If we consider the two differential systems when $A^{2}-1=0$, $B=0$, one of these is

$$
\begin{aligned}
u^{\prime \prime}+\left(\rho^{2}+g\right) u & =0 \\
u^{\prime}(0)+u^{\prime}(1) & =0 \\
u(0)-u(1) & =0 .
\end{aligned}
$$

If we suppose that the equation $g(x)=g(1-x)$ is satisfied almost everywhere on $(0,1)$, the function satisfying the differential system

$$
\begin{array}{r}
u^{\prime \prime}+\left(\rho^{2}+g\right) u=0, \\
u\left(\frac{1}{2}\right)=1, \quad u^{\prime}\left(\frac{1}{2}\right)=0,
\end{array}
$$

is a solution of the irregular system above for all values of $\rho$. Thus it is evident that under appropriate circumstances all values of $\rho$ are characteristic values, while under others there is no characteristic value; in such cases there is no expansion problem.

Having thus obtained a view of some of the peculiarities which can arise in the irregular boundary value problem, we ca'n continue our discussion, under hypotheses which enable us to make more definite assertions. We shall require in all our succeeding work that $g(x)$ be continuous together 
with its derivatives of all orders on the interval $(0,1)$, although we could in many instances lighten this restriction. Then, as we saw in Theorem III' of our previous paper, there exist on the first quadrant of the $\rho$-plane solutions $u_{1}, u_{2}$ of $u^{\prime \prime}+\left(\rho^{2}+g\right) u=0$ with the asymptotic forms

$$
\begin{aligned}
& u_{1}=e^{\rho i x}\left(1+\sum_{l=1}^{l-m} \frac{A_{l 0}(x)}{(\rho i)^{l}}+\frac{E_{10}(x, \rho)}{\rho^{m+1}}\right), \\
& u_{1}^{\prime}=\rho i e^{\rho i x}\left(1+\sum_{l=1}^{l-m} \frac{A_{l 1}(x)}{(\rho i)^{l}}+\frac{E_{11}(x, \rho)}{\rho^{m+1}}\right), \\
& u_{2}=e^{-\rho i x}\left(1+\sum_{l=1}^{l-m} \frac{A_{l 0}(x)}{(-\rho i)^{l}}+\frac{E_{20}(x, \rho)}{\rho^{m+1}}\right), \\
& u_{2}^{\prime}=-\rho i e^{-\rho i x}\left(1+\sum_{l=1}^{l-m} \frac{A_{l 1}(x)}{(-\rho i)^{l}}+\frac{E_{21}(x, \rho)}{\rho^{m+1}}\right),
\end{aligned}
$$

the functions $E$ being uniformly bounded, $0 \leqq x \leqq 1$, for $\rho$ on the first quadrant. In particular, $A_{10}(x)=A_{11}(x)$. Similarly, on the fourth quadrant there exist solutions $u_{1}, u_{2}$ whose asymptotic forms are given by replacing $\rho$ by $-\rho$ in the exponential terms and sums appearing in the forms given above for the first quadrant; the functions $E$ are, of course, not necessarily the same.

THEOREM II. If $u_{1}, u_{2}$ are the functions defined above for the first quadrant, then for any positive integral $m$

$$
\begin{aligned}
D \equiv & \left|\begin{array}{rr}
u_{1}{ }^{\prime}(0)+A u_{1}^{\prime}(1)+B u_{1}(1) & u_{2}^{\prime}(0)+A u_{2}^{\prime}(1)+B u_{2}(1) \\
u_{1}(0)-A u_{1}(1) & u_{2}(0)-A u_{2}(1)
\end{array}\right| \\
= & 2\left(1-A^{2}\right) \rho i\left(1+\frac{E_{1}(\rho)}{\rho^{2}}\right) \\
& +A\left(e^{\rho i}\left(\sum_{l=1}^{l=-m-1} \frac{\alpha_{l}}{\rho^{l}}+\frac{E_{2}(\rho)}{\rho^{m}}\right)+e^{-\rho i}\left(\sum_{l=1}^{l-m-1} \frac{(-1)^{l+1} \alpha_{l}}{\rho^{l}}+\frac{E_{3}(\rho)}{\rho^{m}}\right)\right) \\
+ & B\left(e^{\rho^{i}}\left(1+\sum_{l=1}^{l=m} \frac{\beta_{l}}{\rho^{l}}+\frac{E_{4}(\rho)}{\rho^{m+1}}\right)+e^{-\rho i}\left(1+\sum_{l=1}^{l=m} \frac{(-1)^{l+1} \beta_{l}}{\rho^{l}}+\frac{E_{5}(\rho)}{\rho^{m+1}}\right)\right),
\end{aligned}
$$

$\alpha_{1}, \cdots, \alpha_{m-1}, \beta_{1}, \cdots, \beta_{m}$ being constants, and the functions $E$ being bounded and analytic on the first quadrant. On the fourth quadrant the substitution of the corresponding functions $u_{1}, u_{2}$ gives an analogous formula involving the same numbers $\alpha, \beta$. 
We shall carry through the computations for the first quadrant; by replacing $\rho$ by $-\rho$ in the exponential terms and sums appearing in the expression thus obtained we find the asymptotic form for the fourth quadrant. On expanding the determinant and recalling that

$$
u_{1}^{\prime}(0) u_{2}(0)-u_{1}(0) u_{2}^{\prime}(0)=u_{1}^{\prime}(1) u_{2}(1)-u_{1}(1) u_{2}^{\prime}(1),
$$

we find as the result

$$
\begin{aligned}
\left(1-A^{2}\right)\left(u_{1}^{\prime}(0) u_{2}(0)-u_{1}(0) u_{2}^{\prime}(0)\right)+ & A\left\{\left(\left(u_{1}^{\prime}(1) u_{2}(0)+u_{1}(1) u_{2}^{\prime}(0)\right)\right.\right. \\
& \left.-\left(u_{2}^{\prime}(1) u_{1}(0)+u_{2}(1) u_{1}^{\prime}(0)\right)\right\} \\
+ & B\left(u_{1}(1) u_{2}(0)-u_{1}(0) u_{2}(1)\right) .
\end{aligned}
$$

By direct substitution of the asymptotic forms for $u_{1}, u_{2}$, in which $A_{10}(x)=$ $A_{11}(x)$, as we noted above, we find

$$
\begin{aligned}
& u_{1}^{\prime}(0) u_{2}(0)-u_{1}(0) u_{2}^{\prime}(0)=2 \rho i\left(1+\frac{E_{1}(\rho)}{\rho^{2}}\right), \\
& u_{1}^{\prime}(1) u_{2}(0)+u_{1}(1) u_{2}^{\prime}(0)=e^{\rho i}\left(\sum_{l=1}^{l=m-1} \frac{\alpha_{l}}{\rho^{l}}+\frac{E_{2}(\rho)}{\rho^{m}}\right), \\
& u_{1}(1) u_{2}(0) \quad=e^{\rho i}\left(1+\sum_{l=1}^{l-m} \frac{\beta_{l}}{\rho^{l}}+\frac{E_{4}(\rho)}{\rho^{m+1}}\right)
\end{aligned}
$$

If we neglect the $E$ terms in $u_{1}, u_{2}$, the replacement of $\rho$ by - $\rho$ interchanges $u_{1}, u_{2}$. Hence by replacing $\rho$ by $-\rho$ in the second and third of the asymptotic forms just obtained, we show that

$$
\begin{aligned}
u_{2}^{\prime}(1) u_{1}(0)+u_{2}(1) u_{1}^{\prime}(0) & =e^{-\rho^{i}}\left(\sum_{l=1}^{l-m-1} \frac{(-1)^{l} \alpha_{l}}{\rho^{l}}+\frac{E_{3}(\rho)}{\rho^{m}}\right), \\
u_{2}(1) u_{1}(0) & =e^{-\rho i}\left(1+\sum_{l=1}^{l-m} \frac{(-1)^{l} \beta_{l}}{\rho^{l}}+\frac{E_{b}(\rho)}{\rho^{m+1}}\right) .
\end{aligned}
$$

When the five asymptotic forms are substituted in the expanded determinant, the theorem is obtained.

As a consequence of Theorem II, we lay down

Definition I. The irregular differential system

$$
\begin{aligned}
u^{\prime \prime}+(\lambda+g) u & =0, \lambda=\rho^{2}, \\
u^{\prime}(0)+A u^{\prime}(1)+B u(1) & =0, \\
u(0)-A u(1) & =0,
\end{aligned}
$$


where $g(x)$ is continuous together with its derivatives of all orders, $0 \leqq x \leqq 1$, shall be termed of type 1 if $B \neq 0$; and a system of type $M, M \geqq 2$, if $B=0$ and $\alpha_{M-1}$ is the first of the $\alpha^{\prime}$ s different from zero. If $B=0, \alpha_{1}=0, \alpha_{2}=0$, $\alpha_{3}=0, \cdots$, the system shall be termed of type $\Omega$.

We have found no means of investigating systems of type $\Omega$; that they exist is shown by the examples given above.

In the case of systems of type $M, M=1,2, \ldots$, we can give a complete discussion of the distribution of characteristic values.

THEOREM III. The irregular differential systems of the second order of finite type $M$ have infinitely many characteristic values in $\rho$ when $A^{2}-1=0$. They are distributed asymptotically near the roots of $e^{\rho i}+(-1)^{\mathbf{M}} e^{-\rho i}=0$; for large $|\rho|$ there is one simple characteristic value near each root of this equation.

We recall that the asymptotic forms of $u_{1}, u_{2}$ on a given quadrant are valid also in a sector including the quadrant and bounded by rays parallel to the two axes. Hence this is also true of the asymptotic forms derived from those for $u_{1}, u_{2}$. Thus the characteristic values of $\rho$ on the first quadrant and within a specified distance of it are found as roots of the equation

$$
\begin{aligned}
& B\left(e^{\rho i}[1]-e^{-\rho i}[1]\right)=0, \quad M=1, \\
& A \alpha_{M-1}\left(e^{\rho i}[1]+(-1)^{M} e^{-\rho i}[1]\right) / \rho^{M-1}=0, \quad M \geqq 2,
\end{aligned}
$$

or of

$$
e^{\rho i}[1]+(-1)^{M} e^{-\rho i}[1]=0 \quad(M=1,2, \cdots) .
$$

Similarly the characteristic values on the fourth quadrant and within a specified distance of it are found as the roots of

$$
e^{\rho i}[1]+(-1)^{M} e^{-\rho i}[1]=0 \quad(M=1,2, \cdots) .
$$

The characteristic values on the left half-plane are the negatives of those on the right. Now by methods employed in the discussion of the regular boundary value problem, $n=2$, the roots of the equations $e^{\rho i}[1]+[-1]^{M} e^{-\rho i}=0$ on the two quadrants respectively have the asymptotic distribution described.*

* Birkhoff, these Transactions, vol. 9 (1908), pp. 386-387; Tamarkin, Rendiconti del Circolo Matematico di Palermo, vol. 34 (1912), pp. 353-358; Birkhoff, Rendiconti del Circolo Matematico di Palermo, vol. 36 (1913), pp. 116-118. 
THEOREM IV. The irregular differential systems of the second order of finite type $M$ have infinitely many characteristic values in $\rho$ when $A^{2}-1 \neq 0$. If we write $\mu=2\left(A^{2}-1\right) i / B, M=1$, and $\mu=2\left(A^{2}-1\right) i / A \alpha_{M-1}, M \geqq 2$, the asymptotic distribution of these roots on the right half-plane is as follows:

(1) for $|\rho|$ sufficiently large on the first quadrant, there is one simple characteristic value near each of the points $\rho=r+i s$,

$$
\begin{aligned}
& r=\arccos \frac{(-1)^{M+1} \mu_{1}}{\sqrt{\mu_{1}^{2}+\mu_{2}^{2}}}=\arcsin \frac{(-1)^{M} \mu_{2}}{\sqrt{\mu_{1}^{2}+\mu_{2}^{2}}}, \\
& s=\log \left(\sqrt{\mu_{1}^{2}+\mu_{2}^{2}} r^{M}\right), \quad \mu=\mu_{1}+i \mu_{2} ;
\end{aligned}
$$

(2) for $|\rho|$ sufficiently large on the fourth quadrant, there is one simple characteristic value near each of the points

$$
\begin{aligned}
& r=\arccos \frac{-\mu_{1}}{\sqrt{\mu_{1}^{2}+\mu_{2}^{2}}}=\arcsin \frac{-\mu_{2}}{\sqrt{\mu_{1}^{2}+\mu_{2}^{2}}}, \\
& s=-\log \left(\sqrt{\mu_{1}^{2}+\mu_{2}^{2}} \gamma^{M}\right), \quad \mu=\mu_{1}+i \mu_{2} .
\end{aligned}
$$

The characteristic values on the left half-plane are the negatives of those on the right.

We shall limit our discussion to the characteristic values on the first quadrant; the treatment of those on the fourth quadrant is entirely analogous.

By Theorem II the characteristic values on the first quadrant are found as the roots of

$$
\mu \rho[1]+\frac{e^{\rho i}[1]+(-1)^{M} e^{-\rho i}[1]}{\rho^{M-1}}=0,
$$

or, after multiplication by $(-1)^{M} \rho^{M-1}$, of

$$
e^{-p i}[1]+\mu(-1)^{M} \rho^{M}\left([1]+\frac{e^{p i}[1]}{\mu \rho^{M}}\right)=0
$$

This last equation takes the form

$$
e^{-\rho i}=(-1)^{M+1} \mu \rho^{M}[1]
$$

on the first quadrant.

If there are infinitely many roots $\rho=r+i s$ on the first quadrant, then $\lim _{|\rho|-\infty}(s / r)=0$. To prove this statement it is sufficient to show that there 
exists no positive $\epsilon$ for which infinitely many roots satisfy $s / r \geqq \epsilon$. If such an $\epsilon$ exists, $s \rightarrow \infty$ as $|\rho| \rightarrow \infty$; and for the roots in question

whence

$$
e^{s}(\cos r-i \sin r)=(-1)^{M+1} \mu(r+i s)^{M}[1]
$$

$$
1 \leqq|\mu||[1]|\left|r e^{-s / M}+i s e^{-s / M}\right|^{M} \leqq|\mu||[1]|\left|(s / \epsilon) e^{-s / M}+i s e^{-\imath / M}\right|^{M} \rightarrow 0
$$

as $|\rho| \rightarrow \infty$. The contradiction shows that $\epsilon$ does not exist. Hence

Now we find

$$
\lim _{|\rho| \rightarrow \infty}(s / r)=0
$$

whence

$$
\frac{e^{s}}{|\mu| r^{M}}=|1+i(s / r)|^{M}|[1]|
$$

$$
s-\log \left(\sqrt{\mu_{1}^{2}+\mu_{2}^{2}} r^{M}\right)=M \log |1+i(s / r)|+\log |[1]|=\epsilon_{|\rho|} \rightarrow 0
$$

as $|\rho| \rightarrow \infty$. Using this relation we obtain

$$
\sqrt{\mu_{1}^{2}+\mu_{2}^{2}}(\cos r-i \sin r)=(-1)^{M+1} \mu(1+i(s / r))^{M}[1] e^{-\epsilon|\rho|} \rightarrow(-1)^{M+1} \mu
$$

as $|\rho| \rightarrow \infty$; thus

$$
\begin{aligned}
& r=\arccos \frac{(-1)^{M+1} \mu_{1}}{\sqrt{\mu_{1}^{2}+\mu_{2}^{2}}}+\epsilon_{|\rho|}^{\prime}, \\
& r=\arcsin \frac{(-1)^{M} \mu_{2}}{\sqrt{\mu_{1}^{2}+\mu_{2}^{2}}}+\epsilon_{|\rho|}^{\prime},
\end{aligned}
$$

where $\epsilon_{|\rho|}^{\prime} \rightarrow 0$ as $|\rho| \rightarrow \infty$. In short, if there exist infinitely many characteristic values on the first quadrant, they necessarily lie asymptotically near the points described in (1).

We shall now let $\rho^{\prime}$ be one of the points described under (1). In the function $e^{-\rho i}+(-1)^{M} \mu \rho^{M}[1]$, analytic in $\rho$ on the first quadrant, we replace $\rho$ by $\rho^{\prime}+\xi$; we restrict $\xi$ to the circle $|\xi| \leqq \epsilon^{\prime}<2 \pi$. There results a function analytic in $\xi$,

$$
e^{-\rho^{\prime} i} e^{-i \xi}+(-1)^{M} \mu\left(\rho^{\prime}+\xi\right)^{M}[1] \equiv(-1)^{M+1} \mu r^{\prime} e^{-i \xi}+(-1)^{M} \mu\left(\rho^{\prime}+\xi\right)^{M}[1] .
$$

If we divide by $(-1)^{M} \mu r^{M} \neq 0$ we obtain a new function,

$$
1-e^{-i \xi}+\epsilon\left(\xi, r^{\prime}, s^{\prime}\right),
$$

where $\epsilon\left(\xi, r^{\prime}, s^{\prime}\right)$ is analytic in $\xi,|\xi| \leqq \epsilon^{\prime}$, for all $r^{\prime}, s^{\prime}$, and $\lim _{\left|\rho^{\prime}\right| \rightarrow \infty} \epsilon=0$ uniformly, $|\xi| \leqq \epsilon^{\prime}$. If $\xi$ describes the circle $|\xi|=\epsilon^{\prime \prime} \leqq \epsilon^{\prime}$ the argument of $1-e^{-i \xi}$ changes by $2 \pi$, while $\left|1-e^{-i \xi}\right| \geqq \eta^{\prime}>0$. If we choose $\left|\rho^{\prime}\right|$ so large 
that $\left|\epsilon\left(\xi, r^{\prime}, s^{\prime}\right)\right| \leqq \eta^{\prime} / 2$, the argument of the function $1-e^{-i \xi}+\epsilon\left(\xi, r^{\prime}, s^{\prime}\right)$ also changes by $2 \pi$ when $\xi$ describes the circle $|\xi|=\epsilon^{\prime \prime}$. In other words, $1-e^{-i \xi}+\epsilon$ has a simple zero in the circle $|\xi|=\epsilon^{\prime \prime}$ for all $\rho^{\prime}$ such that $\left|\rho^{\prime}\right| \geqq R^{\prime}$. Thus for large $|\rho|$, there exists one and only one simple characteristic value near each of the points described in (1).

\section{THE IRREGULAR EXPANSION PROBLEMS}

We shall now make a comparative study of the differential systems

$$
\begin{array}{ll}
u^{\prime \prime}+(\lambda+g) u=0, & u^{\prime \prime}+\lambda u=0, \\
u^{\prime}(0)+A u^{\prime}(1)+B u(1)=0, & u^{\prime}(0)-u^{\prime}(1)=0, \\
u(0)-A u(1)=0, & u(0)-u(1)=0 .
\end{array}
$$

The other irregular differential system, adjoint to the first system given here, can be reduced to the form of the first by the substitution $\bar{x}=1-x$ and therefore does not require separate consideration. We assume that the irregular differential system is of finite type. Then there exists for it a Green's function $G(x, y ; \lambda)$; the second differential system has a Green's function $\bar{G}(x, y ; \lambda)$ and gives rise to the expansion problem of Fourier.

We let $\Sigma^{\prime}$ denote the right half-plane for the complex variable $\rho, \lambda=\rho^{2}$, from which the characteristic values of the irregular differential system and of the Fourier system have been removed, the interior of a small circle $\sigma$ of radius $\epsilon$ described about each such value as center being deleted. We denote by $S_{\mathrm{I}}^{\prime}, S_{\mathrm{IV}}^{\prime}$ the parts of $\Sigma^{\prime}$ on the first and fourth quadrants respectively. We denote by $\Gamma$ any circular arc with center at $\rho=0$ and central angle $\pi$, lying on $\Sigma^{\prime}$. The image of $\Gamma$ in the $\lambda$-plane will be a circle $C$; the totality of such circles $C$ forms an infinite set of concentric annular regions none of which contains a characteristic value of either differential system. From the behavior of the characteristic values for large $|\lambda|$, the circles $C_{0}$, $C_{1}, C_{2}, \cdots$, as described in $\S$ II of our previous paper, can be selected from among the circles $C$; this is true simultaneously for the two differential systems we are discussing. Finally, we let $\gamma_{\mathrm{I}}, \gamma_{\mathrm{IV}}$ be the portions of $\Gamma$ on the first and fourth quadrants respectively, while their common radius is $R$.

We shall study the behavior of

$$
\frac{1}{2 \pi i} \frac{\partial^{k}}{\partial x^{k}} \int_{0}^{1} f(y) \int_{C_{\nu}}\left(1-\frac{\lambda^{4}}{\Lambda_{\nu}^{4}}\right)^{k+l} G(x, y ; \lambda) d \lambda d y,
$$

where $f(x)$ is summable on $(0,1), l \geqq M, k=0,1,2, \cdots$, as $\nu \rightarrow \infty$. The meaning of this integral is discussed at length in our preceding paper, $\S \S$ III and IV. 
For this discussion we use Lemmas III, $\mathrm{V}^{\prime}, \mathrm{VI}^{\prime}$ of that paper, as well as Theorem IV. In addition we need two other lemmas.

Lемма I. If $A^{2}-1=0$ in an irregular differential system of order two and type $M$,

$$
\frac{1}{D}= \pm \frac{\rho^{M-1}}{C_{M}\left(e^{\rho i}[1]+(-1)^{M} e^{-\rho i}[1]\right)}
$$

on $S_{\mathrm{I}}^{\prime}$ and $S_{\mathrm{IV}}^{\prime}$ respectively, where $C_{1}=B \neq 0$ and $C_{M}=A \alpha_{M-1} \neq 0, M \geqq 2$.

Furthermore,

$$
\begin{aligned}
& \frac{1}{\left|e^{2 \rho i}[1]+(-1)^{M}[1]\right|} \leqq K, \text { o on } S_{\mathrm{I}}^{\prime} \\
& \frac{1}{\left|[1]+(-1)^{M} e^{-2 \rho i}[1]\right|} \leqq K, \quad \text { on } S_{\mathrm{IV}}^{\prime}
\end{aligned}
$$

The first part of the lemma is virtually a restatement of Theorem II. The second part is proved in exactly the same way as the corresponding facts in the case of regular differential systems.*

Lemma II. If $A^{2}-1 \neq 0$ in an irregular differential system of order two and type $M$,

$$
\frac{1}{D}=\frac{ \pm \rho^{M-1}}{C_{M}\left(\mu \rho^{M}[1]+e^{\rho i}[1]+(-1)^{M} e^{-\rho i}[1]\right)}
$$

where $\mu=2\left(A^{2}-1\right) i / C_{M}$, on $S_{\mathrm{I}}^{\prime}$ and $S_{\mathrm{IV}}^{\prime}$ respectively. Furthermore,

$$
\begin{aligned}
& \frac{1}{\left|e^{2 \rho i}[1]+\mu \rho^{M} e^{\rho i}[1]+(-1)^{M}[1]\right|} \leqq K, \quad \text { son } S_{\mathrm{I}}^{\prime} \\
& \frac{1}{\left|e^{-2 \rho i}(-1)^{M}[1]+\mu \rho^{M} e^{-\rho i}[1]+[1]\right|} \leqq K, \quad \text { o on } S_{\mathrm{IV}}^{\prime} .
\end{aligned}
$$

The first part of the lemma is a restatement of Theorem II.

In order to prove that $\left|e^{2 \rho i}[1]+\mu \rho^{M} e^{\rho i}[1]+(-1)^{M}[1]\right| \geqq 1 / K>0$ on $S_{\mathbf{I}}^{\prime}$ we show that the equality $e^{2 \rho i}[1]+\mu \rho^{M} e^{\rho i}[1]+(-1)^{M}[1]=\eta$, where $|\eta|$ is small, requires that $\rho$ lie near one of the characteristic values on the first quadrant. We let $\rho^{\prime}$ be a value of $\rho$ satisfying this equation, which we can write

$$
e^{\rho^{\prime} i}+\frac{(-1)^{M}[1]-\eta[1]}{\mu \rho^{\prime M}}=0 .
$$

* Birkhoff, Rendiconti del Circolo Matematico di Palermo, vol. 36 (1913), p. 120. 
In the function $e^{2 \rho i}[1]+\mu \rho^{M} e^{\rho i}[1]+(-1)^{M}[1] \equiv \mu \rho^{M} e^{\rho i}[1]+(-1)^{M}[1]$, analytic in $\rho$ on the first quadrant, we write $\rho=\rho^{\prime}+\xi,|\xi| \leqq \epsilon$, where $\epsilon$ is the radius of the circles $\sigma$ described above. We obtain the function

$$
-\frac{(-1)^{M}[1]-\eta[1]}{\rho^{M}}\left(\rho^{\prime}+\xi\right)^{M} e^{i \xi}[1]+(-1)^{M}[1]
$$

analytic in $\xi$. On multiplying it by $e^{-i \xi}(-1)^{M+1} /[1] \neq 0,|\xi| \leqq \epsilon$, the term [1] being the last such term in the preceding expression, we find a function

$$
1-(-1)^{M+1} \eta-e^{-i \xi}+\zeta\left(\xi, \rho^{\prime}, \eta\right)
$$

where $\zeta$ is analytic in $\xi$ for each pair of values $\rho^{\prime}, \eta$, and where also $\lim _{\left|\rho^{\prime}\right| \rightarrow \infty} \zeta\left(\xi, \rho^{\prime}, \eta\right)=0$ uniformly, $|\xi| \leqq \epsilon,|\eta| \leqq H . \quad$ For all $|\eta| \leqq \eta^{\prime}$ where $\eta^{\prime}$ is sufficiently small

$$
\left|1-(-1)^{M+1} \eta-e^{-i \xi}\right| \geqq \delta>0, \quad|\xi|=\epsilon ;
$$

and arg $\left(1-(-1)^{M+1} \eta-e^{-i \xi}\right)$ changes by $2 \pi$ when $\xi$ describes the circle $|\xi|=\epsilon$. We determine $R^{\prime}$ so that

$$
\left|\zeta\left(\xi, \rho^{\prime}, \eta\right)\right| \leqq \frac{\delta}{2}, \quad|\xi|=\epsilon, \quad|\eta| \leqq \eta^{\prime}, \quad\left|\rho^{\prime}\right| \geqq R^{\prime} .
$$

Then arg $\left(1-(-1)^{M+1} \eta-e^{-i \xi}+\zeta\right)$ changes by $2 \pi$ when $\xi$ describes the circle $|\xi|=\epsilon$; and the function itself vanishes in the circle. In other words, there is a characteristic value within distance $\epsilon$ of $\rho=\rho^{\prime}$ if $|\eta| \leqq \eta^{\prime},\left|\rho^{\prime}\right| \geqq R^{\prime}$; and $\rho^{\prime}$ then lies in a circle $\sigma$. Hence, for $|\rho| \geqq R^{\prime}$ on $S_{\mathrm{I}}^{\prime}$ we have

$$
\left|e^{2 \rho i}[1]+\mu \rho^{M} e^{\rho i}[1]+(-1)^{M}[1]\right|>\eta^{\prime},
$$

as we were to show. The statement of the lemma follows at once. Similar reasoning applies on $S_{\text {IV }}^{\prime}$.

We now demonstrate

THEOREM V. For an irregular differential system of the second order of type $M$

$$
\begin{aligned}
& \lim _{R \rightarrow \infty} \int_{0}^{1} f(y) \int_{\gamma_{\mathrm{I}}}\left(1-\frac{\rho^{8}}{R^{8}}\right)^{k+l}\left(2 \rho\left\{\frac{\partial^{k} G}{\partial x^{k}} ; \frac{\partial^{k} G}{\partial x^{k}}\right\}-\left\{F_{\mathrm{I}, k}^{0} ; F_{\mathrm{I}, k}^{1}\right\}\right) d \rho d y=0 \\
& \lim _{R \rightarrow \infty} \int_{0}^{1} f(y) \int_{\gamma_{\mathrm{IV}}}\left(1-\frac{\rho^{8}}{R^{8}}\right)^{k+l}\left(2 \rho\left\{\frac{\partial^{k} G}{\partial x^{k}} ; \frac{\partial^{k} G}{\partial x^{k}}\right\}-\left\{F_{\mathrm{IV}, k}^{0} ; F_{\mathrm{IV}, k}^{1}\right\}\right) d \rho d y=0
\end{aligned}
$$


uniformly, $0<a \leqq x \leqq b<1$, where $k=0,1,2, \cdots, l \geqq M, f(x)$ is summable on $(0,1)$, and

$$
\begin{aligned}
& F_{1, k}^{0}(x, y, \rho) \equiv+\sum_{\rho=0}^{s-k} i^{\rho+1} \rho^{\rho} e^{\rho i(x-y)} \sum_{\alpha+\beta=k-\rho} A_{\alpha k}(x) B_{\beta}(y), \\
& F_{\mathrm{I}, k}^{1}(x, y, \rho) \equiv-\sum_{\rho=0}^{s=k}(-i)^{\rho+1} \rho^{\rho} e^{-\rho i(x-y)} \sum_{\alpha+\beta=k-\rho} A_{\alpha k}(x) B_{\beta}(y), \\
& F_{\mathrm{IV}, k}^{0}(x, y, \rho) \equiv-\sum_{\rho=0}^{\rho-k}(-i)^{\rho+1} \rho^{\rho} e^{-\rho i(x-y)} \sum_{\alpha+\beta=k-\ell} A_{\alpha k}(x) B_{\beta}(y) \\
& F_{\mathrm{IV}, k}^{1}(x, y, \rho) \equiv+\sum_{\ell=0}^{s=k} i^{\rho+1} \rho^{8} e^{\rho i(x-y)} \sum_{\alpha+\beta=k-\rho} A_{\alpha k}(x) B_{\beta}(y) .
\end{aligned}
$$

The functions $A(x), B(y)$ are those defined in our preceding paper in Theorem III' and Lemma XIV. The expression

$$
\frac{1}{2 \pi i} \frac{\partial^{k}}{\partial x^{k}} \int_{0}^{1} f(y) \int_{C_{\nu}}\left(1-\frac{\lambda^{4}}{\Lambda_{\nu}^{4}}\right)^{k+l} G(x, y ; \lambda) d \lambda d y
$$

$k=0,1,2, \cdots, l \geqq M$, is therefore equivalent on any interval $(a, b)$ to $a$ linear combination with coefficients $A_{\alpha k}(x)$ of means of order $k+l$, formed rom the Fourier series and their derived series up to order $k$ for the functions $f(x) B_{0}(x) \equiv f(x), f(x) B_{1}(x), \cdots, f(x) B_{k}(x)$. On any interval $(a, b)$ the expansion problems associated with an irregular differential system of the second order of type $M$ are thus phrased as problems in the theory of Fourier series. In particular

$$
\lim _{\nu \rightarrow \infty} \int_{0}^{1} f(y) \int_{C_{\nu}}\left(1-\frac{\lambda^{4}}{\Lambda_{\nu}^{4}}\right)^{l}(G(x, y ; \lambda)-\bar{G}(x, y ; \lambda)) d \lambda d y=0, l \geqq M,
$$

uniformly, $0<a \leqq x \leqq b<1$.

On putting 
we can write

$$
\begin{gathered}
W_{1}(u) \equiv u^{\prime}(0)+A u^{\prime}(1)+B u(1), \\
W_{2}(u) \equiv u(0)-A u(1), \\
\tau_{1} v_{1}(y)+\tau_{2} v_{2}(y) \equiv \frac{\left|\begin{array}{cc}
u_{1}(y) & u_{2}(y) \\
\tau_{1} & \tau_{2}
\end{array}\right|}{\left|\begin{array}{ll}
u_{1}(y) & u_{2}(y) \\
u_{1}^{\prime}(y) & u_{2}^{\prime}(y)
\end{array}\right|},
\end{gathered}
$$

$$
\begin{gathered}
2 \rho\left\{\frac{\partial^{k} G}{\partial x^{k}} ; \frac{\partial^{k} G}{\partial x^{k}}\right\} \equiv 2 \rho\left\{u_{1}^{(k)}(x) v_{1}(y) ;-u_{2}{ }^{(k)}(x) v_{2}(y)\right\} \\
+2 \rho \frac{\left|\begin{array}{lll}
u_{1}^{(k)}(x) & u_{2}^{(k)}(x) & 0 \\
W_{1}\left(u_{1}\right) & W_{1}\left(u_{2}\right) & +A u_{1}^{\prime}(1) v_{1}(y)+B u_{1}(1) v_{1}(y)-u_{2}^{\prime}(0) v_{2}(y) \\
W_{2}\left(u_{1}\right) & W_{2}\left(u_{2}\right) & -A u_{1}(1) v_{1}(y)-u_{2}(0) v_{2}(y)
\end{array}\right|}{\left|\begin{array}{ll}
W_{1}\left(u_{1}\right) & W_{1}\left(u_{2}\right) \\
W_{2}\left(u_{1}\right) & W_{2}\left(u_{2}\right)
\end{array}\right|} .
\end{gathered}
$$

Employing a familiar notation, we have on $S_{\mathrm{I}}^{\prime}$

$$
\begin{gathered}
2 \rho\left\{u_{1}{ }^{(k)}(x) v_{1}(y) ;-u_{2}^{(k)}(x) v_{2}(y)\right\} \equiv\left\{F_{\mathrm{I}, k}^{0} ; F_{\mathrm{I}, k}^{1}\right\} \\
+\left\{e^{\rho i(x-y)} m_{1}(x, y, \rho) / \rho ; e^{-\rho i(x-y)} m_{2}(x, y, \rho) / \rho\right\} \\
W_{1}\left(u_{1}\right) \equiv \rho i[1]+A \rho i e^{\rho i}[1]+B e^{\rho i}[1] \equiv \rho m(\rho), \\
W_{1}\left(u_{2}\right) \equiv-\rho i[1]-A \rho i e^{-\rho i}[1]+B e^{-\rho i}[1] \equiv \rho m(\rho) e^{-\rho i}, \\
W_{2}\left(u_{1}\right) \equiv[1]-A e^{\rho i}[1] \equiv m(\rho), \\
W_{2}\left(u_{2}\right) \equiv[1]-A e^{-\rho i}[1] \equiv m(\rho) e^{-\rho i}, \\
A u_{1}^{\prime}(1) v_{1}(y)+B u_{1}(1) v_{1}(y)-u_{2}^{\prime}(0) v_{2}(y) \equiv \frac{A}{2} e^{\rho i(1-y)}[1]-\frac{1}{2 \rho} e^{\rho i(1 y}[1] \equiv m(y, \rho), \\
-A u_{1}(1) v_{1}(y)-u_{2}(0) v_{2}(y) \equiv m(y, \rho) / \rho, \\
A u_{1}^{\prime}(1) \int_{\alpha}^{x} v_{1}(y) d y+B u_{1}(1) \int_{\alpha}^{x} v_{1}(y) d y-u_{2}^{\prime}(0) \int_{\alpha}^{x} v_{2}(y) d y \\
-A u_{1}(1) \int_{\alpha}^{x} v_{1}(y) d y-u_{2}(0) \int_{\alpha}^{x} v_{2}(y) d y \equiv \frac{m(x, \rho)}{\rho^{2}}, 0 \leqq \alpha \leqq 1 .
\end{gathered}
$$


The last two results are consequent upon the fact that

$$
\begin{aligned}
e^{\rho i} \int_{\alpha}^{x} v_{1}(y) d y= & \frac{-i}{2 \rho} \int_{\alpha}^{x} e^{\rho i(1-y)}[1] d y=\frac{-i}{2 \rho} \frac{e^{\rho i(1-x)}-e^{\rho i(1-\alpha)}}{-\rho i} \\
& +\frac{-i}{2 \rho} \int_{\alpha}^{x} e^{\rho i(1-y)}[0] d y=m(x, \rho) / \rho^{2} \\
\int_{\alpha}^{x} v_{2}(y) d y= & \frac{i}{2 \rho} \int_{\alpha}^{x} e^{\rho i y}[1] d y=m(x, \rho) / \rho^{2} .
\end{aligned}
$$

Finally

$$
\frac{1}{\left|\begin{array}{ll}
W_{1}\left(u_{1}\right) W_{1}\left(u_{2}\right) \\
W_{2}\left(u_{1}\right) W_{2}\left(u_{2}\right)
\end{array}\right|} \equiv \frac{\rho^{M-1} e^{\rho i}}{C_{M}\left(e^{2 \rho i}[1]+\mu \rho^{M} e^{\rho i}[1]+(-1)^{M}[1]\right)} \equiv \rho^{M-1} m(\rho) e^{\rho i}
$$

by Lemmas I and II. Hence on $S_{\mathrm{I}}^{\prime}$ we find

$$
\begin{aligned}
& 2 \rho\left\{\frac{\partial^{k} G}{\partial x^{k}} ; \frac{\partial^{k} G}{\partial x^{k}}\right\}-\left\{F_{\mathrm{I}, k}^{0} ; F_{\mathrm{I}, k}^{1}\right\} \equiv\left\{e^{\rho i(x-y)} m_{1}(x, y, \rho) / \rho ; e^{-\rho i(x-y)} m_{2}(x, y, \rho) / \rho\right\} \\
&+\rho^{M+k} e^{\rho i x} m_{3}(x, y, \rho)+\rho^{M+k} e^{\rho i(1-x)} m_{4}(x, y, \rho) \\
& \int_{\alpha}^{x}\left(2 \rho\left\{\frac{\partial^{k} G}{\partial x^{k}} ; \frac{\partial^{k} G}{\partial x^{k}}\right\}-\left\{F_{\mathrm{I}, k}^{0} ; F_{\mathrm{I}, k}^{1}\right\}\right) d y \\
& \equiv \int_{\alpha}^{x}\left\{e^{\rho i(x-y)} \frac{m_{1}(x, y, \rho)}{\rho} ; e^{-\rho i(x-y)} \frac{m_{2}(x, y, \rho)}{\rho}\right\} d y \\
& \quad+\rho^{M+k-1} e^{\rho i x} m_{3}(x, \rho)+\rho^{M+k-1} e^{\rho i(1-x)} m_{4}(x, \rho)
\end{aligned}
$$

By Lemmas III, $\mathrm{V}^{\prime}$ of our antecedent paper,

$$
\int_{\gamma_{\mathrm{I}}}\left(1-\frac{\rho^{8}}{R^{8}}\right)^{k+l}\left(2 \rho\left\{\frac{\partial^{k} G}{\partial x^{k}} ; \frac{\partial^{k} G}{\partial x^{k}}\right\}-\left\{F_{\mathrm{I}, k}^{0} ; F_{\mathrm{I}, k}^{1}\right\}\right) d \rho, \quad l \geqq M,
$$

is uniformly bounded, $0<a \leqq x \leqq b<1,0 \leqq y \leqq 1$, for all $\gamma_{\mathrm{I}}$ on $S_{\mathrm{I}}^{\prime}$. Lemmas $\mathrm{V}^{\prime}$ and $\mathrm{VI}^{\prime}$ show that

$$
\lim _{R \rightarrow \infty} \int_{\alpha}^{x} \int_{\gamma_{\mathrm{I}}}\left(1-\frac{\rho^{8}}{R^{8}}\right)^{k+l}\left(2 \rho\left\{\frac{\partial^{k} G}{\partial x^{k}} ; \frac{\partial^{k} G}{\partial x^{k}}\right\}-\left\{F_{\mathrm{I}, k}^{0} ; F_{\mathrm{I}, k}^{1}\right\}\right) d \rho d y=0, l \geqq M,
$$

and also

$$
\lim _{R \rightarrow \infty} \int_{\alpha}^{\beta} \int_{\gamma_{\boldsymbol{I}}}\left(1-\frac{\rho^{8}}{R^{8}}\right)^{k+l}\left(2 \rho\left\{\frac{\partial^{k} G}{\partial x^{k}} ; \frac{\partial^{k} G}{\partial x^{k}}\right\}-\left\{F_{\mathrm{I}, k}^{0} ; F_{\mathrm{I}, k}^{\mathrm{A}}\right\}\right) d \rho d y=0, l \geqq M,
$$


uniformly, $0<a \leqq x \leqq b<1$. An application of the theorem of Lebesgue* quoted as Theorem IV in our previous paper shows that

$$
\lim _{R \rightarrow \infty} \int_{0}^{1} f(y) \int_{\gamma_{\mathrm{I}}}\left(1-\frac{\rho^{8}}{R^{8}}\right)^{k+l}\left(2 \rho\left\{\frac{\partial^{k} G}{\partial x^{k}} ; \frac{\partial^{k} G}{\partial x^{k}}\right\}-\left\{F_{\mathrm{I}, k}^{0} ; F_{\mathrm{I}, k}^{1}\right\}\right) d \rho d y=0, l \geqq M,
$$

uniformly, $0<a \leqq x \leqq b<1$.

Since $u_{1}$ and $u_{2}$ on $S_{\mathrm{I}}^{\prime}$ are changed formally into $u_{1}$ and $u_{2}$ on $\mathrm{S}_{\mathrm{IV}}^{\prime}$ when $\rho$ is replaced by $-\rho$, it is possible to obtain the asymptotic form for

$$
2 \rho\left\{\frac{\partial^{k} G}{\partial x^{k}} ; \frac{\partial^{k} G}{\partial x^{k}}\right\}
$$

on $\mathrm{S}_{\mathrm{I}}^{\prime}$ by replacing $\rho$ by $-\rho$ in that established for $S_{\mathrm{IV}}^{\prime}$. Thus we see almost immediately that

$$
\begin{aligned}
\lim _{R \rightarrow \infty} \int_{0}^{1} f(y) \int_{\gamma_{\mathrm{IV}}}\left(1-\frac{\rho^{8}}{R^{8}}\right)^{k+l}\left(2 \rho\left\{\frac{\partial^{k} G}{\partial x^{k}} ; \frac{\partial^{k} G}{\partial x^{k}}\right\}\right. \\
\left.-\left\{F_{\mathrm{IV}, k}^{0} ; F_{\mathrm{IV}, k}^{1}\right\}\right) d \rho d y=0, \quad l \geqq M,
\end{aligned}
$$

uniformly, $0<a \leqq x \leqq b<1$.

By Lemma XIII of our preceding paper we have

$$
\begin{aligned}
\int_{0}^{1} f(y) \int_{C_{\nu}}\left(1-\frac{\lambda^{4}}{\Lambda_{\nu}{ }^{4}}\right)^{k+l}\left\{\frac{\partial^{k} G}{\partial x^{k}} ; \frac{\partial^{k} G}{\partial x^{k}}\right\} d \lambda d y \\
=\frac{\partial^{k}}{\partial x^{k}} \int_{0}^{1} f(y) \int_{C_{\nu}}\left(1-\frac{\lambda^{4}}{\Lambda_{\nu}^{4}}\right)^{k+l} G d \lambda d y
\end{aligned}
$$

and it is easy to complete the present theorem in a manner analogous to that used for Theorems XXXII and XXXII' of that paper. For $k=0$,

$$
\begin{array}{ll}
F_{\mathrm{I}, 0}^{0}=-i e^{\rho i(x-y)}, & F_{\mathrm{I}, 0}^{1}=-i e^{-\rho i(x-y)}, \\
F_{\mathrm{IV}, 0}^{0}=+i e^{-\rho i(x-y)}, & F_{\mathrm{IV}, 0}^{1}=+i e^{\rho i(x-y)} .
\end{array}
$$

We know that

$$
\begin{aligned}
& \lim _{R \rightarrow \infty} \int_{0}^{1} f(y) \int_{\gamma_{\mathrm{I}}}\left(1-\frac{\rho^{8}}{R^{8}}\right)^{l}\left(\bar{G}-\left\{-i e^{\rho i(x-y)} ;-i e^{-\rho i(x-y)}\right\}\right) d \rho d y=0, l \geqq 0, \\
& \lim _{R \rightarrow \infty} \int_{0}^{1} f(y) \int_{\gamma_{\mathrm{IV}}}\left(1-\frac{\rho^{8}}{R^{8}}\right)^{l}\left(\bar{G}-\left\{i e^{-\rho i(x-y)} ; i e^{\rho i(x-y)}\right\}\right) d \rho d y=0, \quad l \geqq 0,
\end{aligned}
$$

* Lebesgue, Annales de la Faculté des Sciences de Toulouse, (3), vol. 1 (1909), pp. 52-55. 
uniformly, $0<a \leqq x \leqq b<1$, as the evaluation of these limits occurs in the proof sketched for Theorem $\mathrm{IX}^{\prime}$ in our paper on the regular expansion problems. It is immediately evident that

$$
\lim _{\nu \rightarrow \infty} \int_{0}^{1} f(y) \int_{C_{\nu}}\left(1-\frac{\lambda^{4}}{\Lambda_{\nu}^{4}}\right)^{l}(G-\bar{G}) d \lambda d y=0, \quad l \geqq M,
$$

uniformly, $0<a \leqq x \leqq b<1$. This completes the proof of the theorem.

From various known properties of Fourier series and the term-by-term derivative series of Fourier series, we deduce the theorems which follow. The details of proof are strictly analogous to those given under the corresponding theorems of $\S \mathrm{VI}$ of the paper on Birkhoff series.

TheOrem VI. The expansions

$$
\frac{1}{2 \pi i} \frac{\partial^{k}}{\partial x^{k}} \int_{0}^{1} f(y) \int_{C_{\nu}}\left(1-\frac{\lambda^{4}}{\Lambda_{\nu}^{4}}\right)^{k+l} G(x, y ; \lambda) d \lambda d y, \quad l \geqq M,
$$

associated with an irregular differential system of the second order of type $M$ are such that their behavior at $x=x_{0}$ interior to $(0,1)$ is independent of the nature of the summable function $f(x)$ outside an arbitrarily small neighborhood of $x_{0}$.

THEOREM VII. If $f(x)$ is summable, $0 \leqq x \leqq 1$,

$$
\lim _{\nu \rightarrow \infty} \frac{1}{2 \pi i} \int_{0}^{1} f(y) \int_{\mathrm{C}_{\nu}}\left(1-\frac{\lambda^{4}}{\Lambda_{\nu}^{4}}\right)^{k+l} G(x, y ; \lambda) d \lambda d y=f(x)
$$

almost everywhere, $0<x<1$, if $l \geqq M$; if $f(x)$ is continuous ihe convergenie is uniform, $0<a \leqq x \leqq b<1$.

THEOREM VIII. If $\varphi(x)$ is a k-fold integral in the sense of Lebesgue, $0 \leqq x \leqq 1$, then

$$
\lim _{\nu \rightarrow \infty} \frac{1}{2 \pi i} \frac{\partial^{k}}{\partial x^{k}} \int_{0}^{1} \varphi(y) \int_{C_{\nu}}\left(1-\frac{\lambda^{4}}{\Lambda_{\nu}^{4}}\right)^{k+l} G(x, y ; \lambda) d \lambda d y=\varphi^{(k)}(x), l \geqq M,
$$

almost everywhere, $0<x<1$; and if $\varphi^{(k)}(x)$ is continuous the convergence is uniform, $0<a \leqq x \leqq b<1$.

\section{THE IRREGULAR EXPANSION PROBLEMS OF TYPE 1}

If in our differential system we take $g(x) \equiv 0$, there results a special system which is of type 1 or of type $\Omega$. Hence it is only in the case of systems of type $1, B \neq 0$, that we can compare the systems 


$$
\begin{array}{ll}
u^{\prime \prime}+(\lambda+g) u=0, & u^{\prime \prime}+\lambda u=0, \\
u^{\prime}(0)+A u^{\prime}(1)+B u(1)=0, & u^{\prime}(0)+A u^{\prime}(1)+B u(1)=0, \\
u(0)-A u(1)=0, & u(0)-A u(1)=0 .
\end{array}
$$

If we denote by $G, \bar{G}$ the Green's functions for these two differential systems respectively, it is our purpose to study the integral

$$
\frac{1}{2 \pi i} \int_{0}^{1} \int_{C_{\nu}} f(y)(G(x, y ; \lambda)-\bar{G}(x, y ; \lambda)) d \lambda d y .
$$

It will be seen to have the limit zero uniformly, $0<a \leqq x \leqq b<1$, as $\nu \rightarrow \infty$. We then make a special study of the second system, with interesting results.

The notations $\rho, \Sigma^{\prime}, S_{\mathrm{I}}^{\prime}, S_{\mathrm{IV}}^{\prime}, \Gamma, \gamma_{\mathrm{I}}, \gamma_{\mathrm{IV}}, R, C_{\nu}$ have meanings entirely analogous to those in $\S$ III. We do not go into details.

We first prove

LEMma III. On $S_{\mathrm{I}}^{\prime},\left(e^{2 \rho i}+\mu \rho e^{\rho i}+(-1)\right) /\left(e^{2 \rho i}[1]+\mu \rho e^{\rho i}[1]+(-1)[1]\right)=$ [1], and on $S_{\mathrm{IV}}^{\prime},\left(-e^{-2 \rho i}+\mu \rho e^{-\rho i}+1\right) /\left(-e^{-2 \rho i}[1]+\mu \rho e^{-\rho i}[1]+[1]\right)=[1]$.

If $\mu=0$ the lemma is Lemma VIII' of our preceding paper. If $\mu \neq 0$ we recall that in the term $\mu \rho e^{\rho i}[1],[1]=1+E_{1}(\rho) / \rho^{2}$. Consequently

$$
\begin{aligned}
\frac{e^{2 \rho i}+\mu \rho e^{\rho i}+(-1)}{e^{2 \rho i}[1]+\mu \rho e^{\rho i}[1]+(-1)[1]} & =1+\frac{e^{2 \rho i}[0]+\mu \rho e^{\rho i}(1-[1])+[0]}{e^{2 \rho i}[1]+\mu \rho e^{\rho i}[1]+(-1)[1]} \\
& =1+\frac{[0]}{e^{2 \rho i}[1]+\mu \rho e^{\rho i}[1]+(-1)[1]} \\
& =[1],
\end{aligned}
$$

by Lemma II, for all $\rho$ on $S_{\mathrm{I}}^{\prime}$. Similar reasoning applies to the expression on $S_{\text {Iv }}^{\prime}$.

We can now obtain

THEOREM IX. If $f(x)$ is summable on $(0,1)$, then

$$
\lim _{\nu \rightarrow \infty} \int_{0}^{1} f(y) \int_{C_{\nu}}\left(1-\frac{\lambda^{4}}{\Lambda_{\nu}{ }^{4}}\right)^{l}(G(x, y ; \lambda)-\bar{G}(x, y ; \lambda)) d \lambda d y=0, \quad l \geqq 0,
$$

uniformly, $0<a \leqq x \leqq b<1$.

We first show that

$$
\lim _{R \rightarrow \infty} \int_{0}^{1} f(y) \int_{\gamma_{\mathrm{I}}}\left(1-\frac{\rho^{8}}{R^{8}}\right)^{l}(G-\bar{G}) 2 \rho d \rho d y=0, \quad l \geqq 0,
$$

unif ormly, $0<a \leqq x \leqq b<1$; the result holds if $\gamma_{\mathrm{I}}$ is replaced by $\gamma_{\mathrm{IV}}$, by reasoning whose details are now familiar; the theorem follows immediately. 
We find by expanding the formula of Theorem $\mathrm{V}$

$$
\begin{aligned}
& 2 \rho G\left(x, y ; \rho^{2}\right) \equiv 2 \rho\left\{u_{1}(x) v_{1}(y) ;-u_{2}(x) v_{2}(y)\right\} \\
& +\frac{2 \rho e^{\rho i}}{B} \frac{\delta_{11} u_{1}(x) v_{1}(y)+\delta_{12} u_{1}(x) v_{2}(y)+\delta_{21} u_{2}(x) v_{1}(y)+\delta_{22} u_{2}(x) v_{2}(y)}{e^{2 \rho i}[1]+\mu \rho e^{\rho i}[1]-[1]},
\end{aligned}
$$

where

$$
\begin{aligned}
\delta_{11} & =A^{2}\left(u_{1}^{\prime}(1) u_{2}(1)-u_{1}(1) u_{2}{ }^{\prime}(1)\right)-A\left(u_{1}(1) u_{2}{ }^{\prime}(0)+u_{1}{ }^{\prime}(1) u_{2}(0)\right)-B u_{1}(1) u_{2}(0) \\
& =2 A^{2} i \rho[1]-A e^{\rho i}[c]-B e^{\rho i}[1]=2 i \rho\left[A^{2}\right], \\
\delta_{12} & =-A\left(u_{2}(1) u_{2}^{\prime}(0)+u_{2}(0) u_{2}^{\prime}(1)\right)-B u_{2}(0) u_{2}(1) \\
& =-2 A i \rho e^{-\rho i}[1]-B e^{-\rho i}[1]=-2 i \rho e^{-\rho i}[A], \\
\delta_{21} & =A\left(u_{1}(1) u_{1}^{\prime}(0)+u_{1}^{\prime}(1) u_{1}(0)\right)+B u_{1}(0) u_{1}(1) \\
& =2 A i \rho e^{\rho i}[1]+B e^{\rho i}[1]=2 i \rho e^{\rho i}[A], \\
\delta_{22} & =\left(u_{2}(0) u_{1}{ }^{\prime}(0)-u_{2}{ }^{\prime}(0) u_{1}(0)\right)+A\left(u_{1}^{\prime}(1) u_{2}(0)+u_{1}(1) u_{2}{ }^{\prime}(0)\right)+B u_{1}(1) u_{2}(0) \\
& =2 i \rho[1]+A e^{\rho i}[c]+B e^{\rho i}[1]=2 i \rho[1],
\end{aligned}
$$

for $\rho$ on $S_{1}^{\prime}$. Using the asymptotic forms for $u_{1}, u_{2}, v_{1}, v_{2}$ and the result of Lemma III we find

$$
\begin{gathered}
2 \rho G\left(x, y ; \rho^{2}\right)=\left\{-i e^{\rho i(x-y)}[1] ;-i e^{-\rho i(x-y)}[1]\right\} \\
+\frac{1}{B} \frac{e^{\rho i x} e^{\rho i(1-y)}\left[\Delta_{11}\right]+e^{\rho i x} e^{\rho i y}\left[\Delta_{12}\right]+e^{\rho i(1-x)} e^{\rho i(1-y)}\left[\Delta_{21}\right]+e^{\rho i(1-x)} e^{i \rho y}\left[\Delta_{22}\right]}{e^{2 \rho i}+\mu \rho e^{\rho i}-1},
\end{gathered}
$$

where the terms $[\Delta]$ in the numerator are of the forms

$$
\Delta(x, \rho)\left(1+\frac{B_{1}(y)}{\rho i}+\frac{E_{2}(y, \rho)}{\rho^{2}}\right), \Delta(x, \rho)\left(1-\frac{B_{1}(y)}{\rho i}+\frac{E_{2}(y, \rho)}{\rho^{2}}\right),
$$

and where

$$
\begin{aligned}
& \Delta_{11}(x, \rho)=2 \rho\left[A^{2}\right], \\
& \Delta_{12}(x, \rho)=-2 \rho[A], \\
& \Delta_{21}(x, \rho)=2 \rho[A], \\
& \Delta_{22}(x, \rho)=-2 \rho[1] .
\end{aligned}
$$


In particular we have

$$
\begin{aligned}
& 2 \rho \bar{G}\left(x, y ; \rho^{2}\right)=\left\{-i e^{\rho i(x-y)} ;-i e^{-\rho i(x-y)}\right\} \\
& +\frac{1}{B}\left(\frac{1}{e^{2 \rho i}+\mu \rho e^{\rho i}-1}\right)\left(\bar{\Delta}_{11}(x, \rho) e^{\rho i x} e^{\rho i(1-y)}+\bar{\Delta}_{12}(x, \rho) e^{\rho i x} e^{\rho i y}\right. \\
& \left.\quad+\bar{\Delta}_{21}(x, \rho) e^{\rho i(1-x)} e^{\rho i(1-y)}+\bar{\Delta}_{22}(x, \rho) e^{\rho i(1-x)} e^{\rho i y}\right)
\end{aligned}
$$

for $\rho$ on $S_{\mathrm{I}}^{\prime}$.

We see immediately that for $\rho$ on $S_{\mathrm{I}}^{\prime}$

$$
\begin{aligned}
2 \rho\left(G\left(x, y ; \rho^{2}\right)-\bar{G}\left(x, y ; \rho^{2}\right)\right)= & \left\{e^{\rho i(x-y)} m_{1}(x, y, \rho) / \rho ; e^{-\rho i(x-y)} m_{2}(x, y ; \rho) / \rho\right\} \\
& +e^{\rho i x} m_{3}(x, y, \rho)+e^{\rho i(1-x)} m_{4}(x, y ; \rho) .
\end{aligned}
$$

Thus, by Lemmas III and $\mathrm{V}^{\prime}$ of our preceding paper,

$$
\int_{\gamma_{\mathrm{I}}}\left(1-\frac{\rho^{8}}{R^{8}}\right)^{l} 2 \rho(G-\bar{G}) d \rho, \quad l \geqq 0,
$$

is uniformly bounded, $0<a \leqq x \leqq b<1,0 \leqq y \leqq 1$, for all $\gamma_{\mathrm{I}}$ on $S_{\mathrm{I}}^{\prime}$.

To discuss the integral

$$
\int_{\alpha}^{x} \int_{\gamma_{\mathrm{I}}}\left(1-\frac{\rho^{8}}{R^{8}}\right)^{l} 2 \rho(G-\bar{G}) d \rho d y, \quad l \geqq 0,
$$

we observe that

$$
\begin{aligned}
\int_{\alpha}^{x} e^{\rho i(1-y)}\left(1+\frac{B_{1}(y)}{\rho i}+\frac{E_{1}}{\rho^{2}}\right) d y= & \frac{e^{\rho i(1-\alpha)}-e^{\rho i(1-x)}}{\rho i} \\
& +\frac{B_{1}(x) e^{\rho i(1-x)}-B_{1}(\alpha) e^{\rho i(1-\alpha)}}{\rho^{2}} \\
& +\int_{\alpha}^{x} e^{\rho i(1-y)} \frac{E_{1}-B_{1}{ }^{\prime}(y)}{\rho^{2}} d y \\
= & \frac{e^{\rho i(1-\alpha)}-e^{\rho i(1-x)}}{\rho i}+\frac{m(x, \alpha, \rho)}{\rho^{2}}, \\
\int_{\alpha}^{x} e^{\rho i y}\left(1-\frac{B_{1}(y)}{\rho i}+\frac{E_{2}}{\rho^{2}}\right) d y= & \frac{e^{\rho i x}-e^{\rho i \alpha}}{\rho i}+\frac{m(x, \alpha, \rho)}{\rho^{2}} .
\end{aligned}
$$


Thus we have

$$
\begin{aligned}
\int_{\alpha}^{x} 2 \rho(G-\bar{G}) d y & =\int_{\alpha}^{x}\left\{e^{\rho i(x-y)} m_{1}(x, y, \rho) / \rho ; e^{-\rho i(x-y)} m_{2}(x, y, \rho) / \rho\right\} d y \\
& +\frac{1}{B}\left(\frac{1}{e^{2 \rho i}+\mu \rho e^{\rho i}-1}\right)\left(e ^ { \rho i x } \int _ { \alpha } ^ { x } \left(e^{\rho i(1-y)}\left[\Delta_{11}\right]\right.\right. \\
& \left.-\bar{\Delta}_{11}(x, \rho) e^{\rho i(1-y)}\right) d y+\cdots+e^{\rho i(1-x)} \int_{\alpha}^{x}\left(e^{\rho i y}\left[\Delta_{22}\right]\right. \\
& \left.\left.-\bar{\Delta}_{22}(x, \rho) e^{\rho i y}\right) d y\right)
\end{aligned}
$$

where the coefficients of $e^{\rho i x}, e^{\rho i(1-x)}$ are of the form $m_{3}(x, \alpha, \rho) / \rho, m_{\mathbf{4}}(x, \alpha, \rho) / \rho$ respectively; we compute, for instance,

$$
\begin{array}{r}
\int_{\alpha}^{x}\left(e^{\rho i(1-y)}\left[\Delta_{11}\right]-\bar{\Delta}_{11}(x, \rho) e^{\rho i(1-y)}\right) d y=\left(\Delta_{11}(x, \rho)-\bar{\Delta}_{11}(x, \rho)\right) \frac{e^{\rho i(1-\alpha)}-e^{\rho i(1-x)}}{\rho i} \\
+\frac{\Delta_{11}(x, \rho) m_{1}(x, \alpha, \rho)}{\rho^{2}}+\frac{\bar{\Delta}_{11}(x, \rho) m_{2}(x, \alpha, \rho)}{\rho^{2}}=m(x, \alpha, \rho) / \rho
\end{array}
$$

Hence we find

$$
\begin{aligned}
\int_{\alpha}^{x} 2 \rho(G-\bar{G}) d y= & \int_{\alpha}^{x}\left\{e^{\rho i(x-y)} m_{1}(x, y, \rho) / \rho ; e^{-\rho i(x-y)} m_{2}(x, y, \rho) / \rho\right\} d y \\
& +e^{\rho i x} m_{3}(x, \alpha, \rho) / \rho+e^{\rho i(1-x)} m_{4}(x, \alpha, \rho)
\end{aligned}
$$

Lemmas $\mathrm{V}^{\prime}$ and $\mathrm{VI}^{\prime}$ of our antecedent paper show that

$$
\int_{\alpha}^{x} \int_{\gamma_{\mathrm{I}}}\left(1-\frac{\rho^{8}}{R^{8}}\right)^{l} 2 \rho\left(G\left(x, y ; \rho^{2}\right)-\bar{G}\left(x, y ; \rho^{2}\right)\right) d \rho d y \rightarrow 0, \quad l \geqq 0,
$$

as $R \rightarrow \infty$, uniformly, $0<a \leqq x \leqq b<1$.

The reasoning then follows the usual channels until we have

$$
\lim _{\mathrm{R} \rightarrow \infty} \int_{0}^{1} f(y) \int_{\gamma_{\mathrm{I}}}\left(1-\frac{\rho^{8}}{R^{8}}\right)^{l} 2 \rho\left(G\left(x, y ; \rho^{2}\right)-\bar{G}\left(x, y ; \rho^{2}\right)\right) d \rho d y=0, \quad l \geqq 0,
$$

uniformly, $0<a \leqq x \leqq b<1$; and

$$
\lim _{\nu \rightarrow \infty} \int_{0}^{1} f(y) \int_{C_{\nu}}\left(1-\frac{\lambda^{4}}{\Lambda_{\nu}^{4}}\right)^{l}(G(x, y ; \lambda)-\bar{G}(x, y ; \lambda)) d \lambda d y=0, \quad l \geqq 0,
$$

uniformly, $0<a \leqq x \leqq b<1$. The proof is thus completed. 
Because of the result just obtained, it is of interest to study the differential system

$$
\begin{array}{ll}
u^{\prime \prime}+\lambda u=0, \quad \lambda=\rho^{2}, & 0 \leqq x \leqq 1, \\
u^{\prime}(0)+A u^{\prime}(1)+B u(1)=0, & B \neq 0, \\
u(0)-A u(1)=0 . &
\end{array}
$$

We find that there are three cases to consider according as $A=-1, A=+1$, $A^{2}-1 \neq 0$; we shall call them Cases I, II, III, and take them up in order.

In Case $I$ the differential system and its adjoint are respectively

$$
\begin{array}{ll}
u^{\prime \prime}+\rho^{2} u=0, & v^{\prime \prime}+\rho^{2} v=0, \\
u^{\prime}(0)-u^{\prime}(1)+B u(1)=0, & v^{\prime}(0)+v^{\prime}(1)-B v(0)=0, \\
u(0)+u(1)=0, & v(0)-v(1)=0 .
\end{array}
$$

The characteristic values of $\rho$ are found from the equation $e^{\rho i}-e^{-\rho i}=0$; in fact, they are all simple and are given by $\rho=k \pi, k= \pm 1, \pm 2, \cdots$. We need consider only positive values of $k$. The corresponding solutions of the differential system are then

$$
\begin{aligned}
& u_{1}=\sqrt{2} \sin \pi x+\frac{2 \sqrt{2}}{B} \pi \cos \pi x, v_{1}=\sqrt{2} \sin \pi x, \\
& u_{2}=\sqrt{2} \sin 2 \pi x, \quad v_{2}=\sqrt{2} \sin 2 \pi x+\frac{2 \sqrt{2}}{B} 2 \pi \cos 2 \pi x, \\
& u_{2 m}=\sqrt{2} \sin 2 m \pi x, \quad v_{2 m}=\sqrt{2} \sin 2 m \pi x+\frac{2 \sqrt{2}}{B} 2 m \pi \cos 2 m \pi x, \\
& u_{2 m+1}=\sqrt{2} \sin (2 m+1) \pi x+\frac{2 \sqrt{2}}{B}(2 m+1) \pi \cos (2 m+1) \pi x, \\
& v_{2 m+1}=\sqrt{2} \sin (2 m+1) \pi x
\end{aligned}
$$

as can be verified by direct substitution. For these solutions we find

$$
\int_{0}^{1} u_{i} v_{k} d x=\left\{\begin{array}{ll}
0, & i \neq k \\
1, & i=k
\end{array}\right\} .
$$

The expansion problem is therefore that of representing an arbitrary summable function in terms of the infinite series

$$
\sum_{k=1}^{k=\infty} a_{k} v k_{k}, \quad a_{k}=\int_{0}^{1} f v_{k} d x .
$$


The sum of the first $N$ terms of this series can be written

$$
\sum_{k=1}^{k=N} a_{k} u_{k}=\sum_{k=1}^{k=N} a_{k}^{\prime} \sqrt{2} \sin k \pi x+\frac{4}{B} \sum_{k=1}^{k=N} \frac{d}{d x}\left(a_{k^{\prime \prime}} \cos k \pi x+b_{k^{\prime \prime}} \sin k \pi x\right),
$$

where

$$
\begin{aligned}
a_{k}{ }^{\prime} & =\sqrt{2} \int_{0}^{1} f(y) \sin k \pi y d y, \\
a_{2 m}^{\prime \prime} & =-\int_{0}^{1} f(y) \cos 2 m \pi y d y, \\
a_{2 m+1}^{\prime \prime} & =0, \\
b_{2 m}^{\prime \prime} & =0, \\
b_{2 m+1}^{\prime \prime} & =\int_{0}^{1} f(y) \sin (2 m+1) \pi y d y .
\end{aligned}
$$

If $F_{1}(x)$ is defined for the interval $(0,2)$ by the equations

$$
\begin{aligned}
& F_{1}(x)=0, \quad 0 \leqq x \leqq 1 \\
& F_{1}(x)=\frac{f(x-1)+f(2-x)}{2}, \quad 1 \leqq x \leqq 2,
\end{aligned}
$$

its expansion in terms of Fourier series on the interval $(0,2)$ is given by

$$
A_{0}+\sum_{k=1}^{k=\infty}\left(A_{k} \cos k \pi x+B_{k} \sin k \pi x\right)
$$

where

$$
\begin{aligned}
& A_{0}=\frac{1}{2} \int_{0}^{2} F_{1}(y) d y, \\
& A_{k}=\int_{0}^{2} F_{1}(y) \cos k \pi y d y=\int_{0}^{1} \frac{f(y)+f(1-y)}{2} \cos k \pi(y+1) d y=a_{k^{\prime \prime}}, \\
& B_{k}=\int_{0}^{2} F_{1}(y) \sin k \pi y d y=\int_{0}^{1} \frac{1(y)+f(1-y)}{2} \sin k \pi(y+1) d y=b_{k^{\prime \prime}},
\end{aligned}
$$

by a series of obvious manipulations. In other words, the expression

$$
0+\sum_{k=1}^{k=N} \frac{d}{d x}\left(a_{k^{\prime \prime}} \cos k \pi x+b_{k}^{\prime \prime} \sin k \pi x\right)
$$

is the sum of the first $2 N+1$ terms of the term-by-term derived series of the Fourier series for $F_{1}(x)$, a function identically zero, $0 \leqq x<1$. We recall at this point some of the theorems concerning the derived series of Fourier 
series. In particular the Cesàro mean of order $l>0$ for the present series converges uniformly to zero, $0<a \leqq x \leqq b<1$. $^{*}$ The term $\Sigma a_{k}{ }^{\prime} \sqrt{2} \sin k \pi x$ is the ordinary sine series on $(0,1)$.

Thus from Theorem VI of our preceding paper and Theorem IX of the present one we have

THEOREM X. If $G(x, y ; \lambda)$ is the Green's function for an irregular differential system of the second order of type 1 , Case $\mathrm{I}$, and if $f(x)$ is summable on $(0,1)$, then the expression

$$
\frac{1}{2 \pi i} \int_{0}^{1} f(y) \int_{C_{\nu}}\left(1-\frac{\lambda^{4}}{\Lambda_{\nu}^{4}}\right)^{l} G(x, y ; \lambda) d \lambda d y, \quad l \geqq 0,
$$

is equivalent on any interval $(a, b)$ completely interior to $(0,1)$ to a sum of means of order $l$ formed from the sine series on $(0,1)$ for $f(x)$ and from the derived series of the Fourier series on $(0,2)$ for $F_{1}(x)$, where $F_{1}(x)$ is the function defined above. In consequence,

$$
\lim _{\nu \rightarrow \infty} \frac{1}{2 \pi i} \int_{0}^{1} f(y) \int_{C_{\nu}}\left(1-\frac{\lambda^{4}}{\Lambda_{\nu}^{4}}\right)^{l} G(x, y ; \lambda) d \lambda d y=f(x), \quad l>0,
$$

almost everywhere, $0<x<1$; the convergence is uniform on $(a, b)$ if $f(x)$ is continuous on $(0,1)$.

This theorem is stronger in the case $M=1, A=-1$, than Theorem V; it has also the advantage of revealing clearly the precise nature of the irregularity in the expansion problem.

In Case II, $A=+1$, we obtain entirely similar results. The differential system can be solved and the formal series set up as before. It is found that the expansion of an arbitrary summable function $f(x)$ is representable as the sum of the sine series on $(0,1)$ for $f(x)$ and the term-by-term derived series of the Fourier series on $(0,2)$ for $F_{2}(x)$, where

$$
\begin{array}{ll}
F_{2}(x)=0, & 0 \leqq x \leqq 1, \\
F_{2}(x)=\frac{f(2-x)-f(x-1)}{2}, & 1 \leqq x \leqq 2 .
\end{array}
$$

It is therefore possible to state the following theorem.

- W. H. Young, Proceedings of the London Mathematical Society, (2), vol. 13 (1914), pp. 13-28; also \& VI of our preceding paper. 
TheOREM XI. If $G(x, y ; \lambda)$ is the Green's function for an irregular differential system of the second order of type 1, Case II, then the expression

$$
\frac{1}{2 \pi i} \int_{0}^{1} f(y) \int_{C_{\nu}}\left(1-\frac{\lambda^{4}}{\Lambda^{4}}\right)^{l} G(x, y ; \lambda) d \lambda d y, \quad l \geqq 0,
$$

formed for any summable funciion $f(x)$, is equivalent on any interval $(a, b)$ completely interior to $(0,1)$ to a sum of means of order $l$ formed from the sine series for $f(x)$ on $(0,1)$ and from the derived series of the Fourier series for $F_{2}(x)$ on $(0,2)$, where $F_{2}(x)$ is the function defined above. In consequence

$$
\lim _{\nu \rightarrow \infty} \frac{1}{2 \pi i} \int_{0}^{1} f(y) \int_{C_{\nu}}\left(1-\frac{\lambda^{4}}{\Lambda_{\nu}^{4}}\right)^{l} G(x, y ; \lambda) d \lambda d y=f(x), \quad l>0,
$$

almost everywhere, $0<x<1$; the convergence is uniform on $(a, b)$ if $f(x)$ is continuous on $(0,1)$.

To discuss Case III, $A^{2}-1 \neq 0$, we first prove

Lemma IV. If $\rho=r+i s, 0<C<|\mu|$, then

$$
\left|\frac{1}{e^{2 \rho i}+\mu \rho e^{\rho i}-1}\right| \leqq K e^{s} / r, \quad 0 \leqq s \leqq \log C_{r}, \quad r \geqq 0,
$$

for $|\rho|$ sufficiently large; and

$$
\left|\frac{1}{1+\mu \rho e^{-\rho i}-e^{-2 \rho i}}\right| \leqq K e^{-s} / r, \quad-\log C r \leqq s \leqq 0, \quad r \geqq 0,
$$

for $|\rho|$ sufficiently large.

We take up the first inequality only, the other being treated similarly. We have

$$
e^{2 \rho i}+\mu \rho e^{\rho i}-1=\mu \rho e^{\rho i}[1]-1
$$

Hence

$$
\begin{aligned}
\mid e^{2 \rho i} & +\mu \rho e^{\rho i}-1|=| \mu \rho e^{\rho i}[1]-1 \mid \\
& =r e^{-s}\left|\mu\left(1+\frac{i s}{r}\right)(\cos r+i \sin r)[1]-\frac{e^{s}}{r}\right| \\
& \geqq r e^{-s}\left(|\mu|\left|1+\frac{i s}{r}\right||[1]|-C\right)>\frac{r e^{-s}}{K}, 0 \leqq s \leqq \log C r, r \geqq 0,
\end{aligned}
$$

for $|\rho|$ sufficiently large, since the term in the last parenthesis has the positive limit $|\mu|-C$ as $|\rho| \rightarrow \infty$, uniformly for the range of $s$ considered. 
We can now prove

TheOREM XII. If $G(x, y ; \lambda)$ is the Green's function for an irregular differential system of the second order of type 1 , Case III; $\bar{G}(x, y ; \lambda)$ is the Green's function for the Fourier differential system of the second order

$$
\begin{aligned}
& u^{\prime \prime}+\lambda u=0 \\
& u^{\prime}(0)-u^{\prime}(1)=0 \\
& u(0)-u(1)=0
\end{aligned}
$$

and $\varphi(x)$ is of bounded variation on $(0,1)$; then

$$
\lim _{\nu \rightarrow \infty} \frac{1}{2 \pi i} \int_{0}^{1} \varphi(y) \int_{C_{\nu}}\left(1-\frac{\lambda^{4}}{\Lambda_{\nu}^{4}}\right)^{l}(G(x, y ; \lambda)-\bar{G}(x, y ; \lambda)) d \lambda d y=0, \quad l \geqq 0,
$$

uniformly, $0<a \leqq x \leqq b<1$. Thus

$$
\lim _{\nu \rightarrow \infty} \frac{1}{2 \pi i} \int_{0}^{1} \varphi(y) \int_{C_{\nu}}\left(1-\frac{\lambda^{4}}{\Lambda_{\nu}{ }^{4}}\right)^{l} G(x, y ; \lambda) d \lambda d y=\frac{\varphi(x+0)+\varphi(x-0)}{2}, l \geqq 0,
$$

$0<x<1$; the convergence is uniform on $(a, b)$ if $\varphi(x)$ is continuous on $(0,1)$.

It suffices to prove the theorem for a monotone function $\varphi(x)$ and, by Theorem IX, the Green's function for the system

$$
\begin{aligned}
& u^{\prime \prime}+\lambda u=0, \\
& u^{\prime}(0)+A u^{\prime}(1)+B u(1)=0, \\
& u(0)-A u(1)=0,
\end{aligned}
$$

We apply the second law of the mean for integrals to the expressions

$$
\begin{gathered}
\int_{0}^{1} \varphi(y) e^{\rho i(1-y)} d y=\varphi(+0) \int_{0}^{\xi_{1}} e^{\rho i(1-y)} d y+\varphi(1-0) \int_{\xi_{1}}^{1} e^{\rho i(1-y)} d y=\frac{m(\rho)}{\rho}, \\
\int_{0}^{1} \varphi(y) e^{\rho i y} d y=\varphi(+0) \int_{0}^{\xi_{2}} e^{\rho i y} d y+\varphi(1-0) \int_{\xi_{2}}^{1} e^{\rho i y} d y=\frac{m(\rho)}{\rho} .
\end{gathered}
$$

On substituting these results in the expression for $G$ given in Theorem IX, we find

$$
\begin{aligned}
\int_{0}^{1} \varphi(y) 2 \rho & \left(1-\frac{\rho^{8}}{R^{8}}\right)^{l} G\left(x, y ; \rho^{2}\right) d y=\int_{0}^{1} \varphi(y)\left\{-i e^{\rho i(x-y)} ;\right. \\
& \left.-i e^{-\rho i(x-y)}\right\}\left(1-\frac{\rho^{8}}{R^{8}}\right)^{l} d y+\frac{e^{\rho i x} m_{1}(\rho)+e^{\rho i(1-x)} m_{2}(\rho)}{e^{2 \rho i}+\mu \rho e^{\rho i}-1}, \quad l \geqq 0 .
\end{aligned}
$$


We now show that

$$
\lim _{R \rightarrow \infty} \int_{\gamma_{\mathrm{I}}} \frac{e^{\rho i x} m_{1}(\rho)+e^{\rho i(1-x)} m_{2}(\rho)}{e^{2 \rho i}+\mu \rho e^{\rho i}-1} d \rho=0,
$$

uniformly, $0<a \leqq x \leqq b<1$. To do this we write $\rho=R e^{i \theta}, 0 \leqq \theta \leqq \pi / 2$, on $\gamma_{\mathrm{I}}$, and then investigate the integrals for $0 \leqq \theta \leqq \theta_{1}, \theta_{1} \leqq \theta \leqq \pi / 4, \pi / 4 \leqq \theta \leqq \pi / 2$, where $\theta_{1}$ satisfies the equation $R \sin \theta_{1}=\log C R \sin \theta_{1}$. That $\theta_{1}$ exists and is unique is seen very readily; it is the argument of the point of intersection of $\gamma_{\mathrm{I}}$ and the curve $C r=e^{s}$. Then we have, if $\delta>0$ is the lesser of $a$, $1-b$, and if $\left|m_{1}\right| \leqq M / 2,\left|m_{2}\right| \leqq M / 2$, because of Lemma IV,

$$
\begin{gathered}
\left|\int_{0}^{\theta_{1}} \frac{e^{\rho i x} m_{1}+e^{\rho i(1-x)} m_{2}}{e^{2 \rho i}+\mu \rho e^{\rho i}-1} R e^{i \theta} d \theta\right| \leqq M K \int_{0}^{\theta_{1}} \frac{e^{R(1-\delta) \sin \theta}}{\cos \theta d \theta} \\
\leqq \frac{M K}{\cos ^{2} \theta_{1}} \int_{0}^{\theta 1} e^{R(1-\delta) \sin \theta} \cos \theta d \theta=\frac{M K}{\cos ^{2} \theta_{1}} \frac{e^{R(1-\delta)}-1}{R(1-\delta)} \\
=\frac{M K}{\cos ^{2} \theta_{1}} \frac{\left(C R \cos \theta_{1}\right)^{1-\delta}-1}{R(1-\delta)} \rightarrow 0, \quad \theta_{1} \rightarrow 0,
\end{gathered}
$$

as $R \rightarrow \infty$. Again, by Lemma II,

$$
\begin{aligned}
& \left|\int_{\theta_{1}}^{\pi / 4} \frac{e^{\rho i x} m_{1}+e^{\rho i(1-x)} m_{2}}{e^{2 \rho i}+\mu \rho e^{\rho i}-1} R e^{i \theta} d \theta\right| \leqq M K \int_{\theta_{1}}^{\pi / 4} e^{-\delta R \sin \theta} R d \theta \\
\leqq & \frac{M K}{\cos (\pi / 4)} \int_{\theta_{1}}^{\pi / 4} e^{-\delta R \sin \theta} R \cos \theta d \theta=\frac{M K}{\delta \cos (\pi / 4)}\left(e^{-R \delta \sin (\pi / 4)}-e^{-\delta R \sin \theta_{2}}\right) \\
= & \frac{M K}{\delta \cos (\pi / 4)}\left(e^{-R \delta \sin (\pi / 4)}-\left(C R \cos \theta_{1}\right)^{-\delta}\right) \rightarrow 0
\end{aligned}
$$

as $R \rightarrow \infty$. Finally

$$
\left|\int_{\pi / 4}^{\pi / 2} \frac{e^{\rho i x} m_{1}+e^{\rho i(1-x)} m_{2}}{e^{2 \rho i}+\mu \rho e^{\rho i}-1} R e^{i \theta} d \theta\right| \leqq M K \int_{\pi / 4}^{\pi / 2} R e^{-\delta R \sin (\pi / 4)} d \theta \rightarrow 0
$$

as $R \rightarrow \infty$. We have established the desired result.

It follows at once that

$$
\lim _{R \rightarrow \infty} \int_{0}^{1} \varphi(y) \int_{\gamma_{1}}\left(1-\frac{\rho^{8}}{R^{8}}\right)^{l}\left(2 \rho G-\left\{-i e^{\rho i(x-y)} ;-i e^{-\rho i(x-y)}\right\}\right) d \rho d y=0, l \geqq 0
$$


uniformly, $0<a \leqq x \leqq b<1$. We know that we can replace $G$ by $\bar{G}$, the Green's function for the differential system

$$
\begin{aligned}
& u^{\prime \prime}+\lambda u=0, \\
& u^{\prime}(0)-u^{\prime}(1)=0, \\
& u(0)-u(1)=0,
\end{aligned}
$$

in this expression. Thus

$$
\lim _{R \rightarrow \infty} \int_{0}^{1} \varphi(y) \int_{\gamma_{1}}\left(1-\frac{\rho^{8}}{R^{8}}\right)^{l} 2 \rho(G-\bar{G}) d \rho d y=0, \quad l \geqq 0,
$$

and

$$
\lim _{\nu \rightarrow \infty} \int_{0}^{1} \varphi(y) \int_{C_{\nu}}\left(1-\frac{\lambda^{4}}{\Lambda_{\nu}^{4}}\right)^{l}(G(x, y ; \lambda)-\bar{G}(x, y ; \lambda)) d \lambda d y=0, \quad l \geqq 0,
$$

uniformly, $0<a \leqq x \leqq b<1$. The remainder of the theorem follows at once.

The differential system

$$
\begin{array}{ll}
u^{\prime \prime}+\lambda u=0, & 0 \leqq x \leqq 1, \\
u^{\prime}(0)+A u^{\prime}(1)+B u(1)=0, & \\
u(0)-A u(1)=0, & B \neq 0,
\end{array}
$$

was so considered in Cases I and II that knowledge concerning the behavior at $x=0$ and at $x=1$ of the expansions associated with them was contained in the theorems proved; these two points are obviously points at which the irregularity of the differential system renders the expansions especially peculiar. In Case III we must study the expansions at these points separately. We take the characteristic equation in the form $\sin \rho=\bar{\mu} \rho$ instead of $e^{\rho i}+\mu \rho-e^{-\rho i}=0$. For large $|\rho|$ the characteristic values corresponding to roots of this equation are all simple. We denote them by $\rho_{K}, \rho_{K_{+1}}$, $\rho_{\mathbf{K}+2}, \cdots$, where $\left|\rho_{k+1}\right| \geqq\left|\rho_{k}\right|, k=K, K+1, \cdots$. Since $A^{2}-1 \neq 0$, the functions

and

$$
U_{k}=\sin \rho_{k} x+A \sin \rho_{k}(1-x)
$$

$$
V_{k}=A \sin \rho_{k} x-\sin \rho_{k}(1-x) \quad(k=K, K+1, \cdots),
$$

satisfy the differential system and its adjoint for $\rho=\rho_{k}$. 
Then

$$
\begin{aligned}
& \int_{0}^{1} U_{i} V_{k} d y= 0, \quad i \neq k, \quad i, k \geqq K \\
& \int_{0}^{1} U_{k} V_{k} d y=A \int_{0}^{1}\left(\sin ^{2} \rho_{k} y-\sin ^{2} \rho_{k}(1-y)\right) d y \\
& \quad+\left(A^{2}-1\right) \int_{0}^{1} \sin \rho_{k} y \sin \rho_{k}(1-y) d y \\
&=\frac{A^{2}-1}{2} \int_{0}^{1}\left(\cos \rho_{k}(2 y-1)-\cos \rho_{k}\right) d y \\
&=\frac{1-A^{2}}{2}\left(\cos \rho_{k}-\frac{\sin \rho_{k}}{\rho_{k}}\right)=\frac{1-A^{2}}{2}\left(\cos \rho_{k}-\bar{\mu}\right) .
\end{aligned}
$$

The expansion of an arbitrary summable function thus takes the form

$$
\sum_{k=1}^{k-K} \int_{0}^{1} f(y) R_{k}(x, y) d y+\sum_{k=K}^{k-\infty} \frac{2}{1-A^{2}} \frac{U_{k}(x)}{\cos \rho_{k}-\bar{\mu}} \int_{0}^{1} f V_{k} d y .
$$

In the case that $A=0$, the boundary condition $u(0)=0$ shows us that for $x=0$ this expansion converges to zero; hence we consider the case $x=0$ only when $A \neq 0$. We shall discuss the convergence at $x=0, x=1$ of the above series for a function $f(x)$ continuous with its first three derivatives on $(0,1)$. It is unnecessary to treat the first $K-1$ terms for our purpose. We have at once

$$
\begin{aligned}
\int_{0}^{1} f V_{k} d y= & -f(y)\left(A \cos \rho_{k} y+\cos \rho_{k}(1-y)\right) /\left.\rho_{k}\right|_{y=0} ^{y-1} \\
& +f^{\prime}(y)\left(A \sin \rho_{k} y-\sin \rho_{k}(1-y)\right) /\left.\rho_{k}^{2}\right|_{y=0} ^{y=1} \\
& +f^{\prime \prime}(y)\left(A \cos \rho_{k} y+\cos \rho_{k}(1-y)\right) /\left.\rho_{k}^{3}\right|_{y=0} ^{y=1} \\
& -\frac{1}{\rho_{k}^{3}} \int_{0^{3}}^{1} f^{\prime \prime \prime}(y)\left(A \cos \rho_{k} y+\cos \rho_{k}(1-y)\right) d y .
\end{aligned}
$$

The first two terms combine as $\left(\alpha \cos \rho_{k}+\beta\right) / \rho_{k}$ where $\alpha, \beta$ are constants; $\alpha$, in particular, is the expression $f(0)-A f(1)$. Since we have 


$$
\begin{aligned}
\left|\frac{\cos \rho_{k} \xi}{\rho_{k}}\right|= & \frac{1}{\left|\rho_{k}\right|}\left(\cosh ^{2} s_{k} \xi \cos ^{2} r_{k} \xi+\sinh ^{2} s_{k} \xi \sin ^{2} r_{k} \xi\right)^{1 / 2} \\
= & \frac{1}{\left|\rho_{k}\right|}\left(\cosh ^{2} s_{k} \xi-\sin ^{2} r_{k} \xi\right)^{1 / 2} \leqq \cosh s_{k} \xi /\left|\rho_{k}\right| \\
\leqq & \cosh s_{k} /\left|\rho_{k}\right| \leqq Q
\end{aligned}
$$

for $0 \leqq \xi \leqq 1, \rho_{k}=r_{k}+i s_{k}, k=K, K+1, \cdots$, by the results of Theorem IV, we can write the sum of the last two terms as $m_{k} / \rho_{k}^{2}$ where $m_{k}$ is bounded, $k=K, K+1, \cdots$. We have, then, to consider the series

$$
\sum_{k=K}^{k=\infty} \frac{2}{1-A^{2}} \frac{U_{k}(x)}{\rho_{k}}\left(\frac{\alpha \cos \rho_{k}+\beta}{\cos \rho_{k}-\bar{\mu}}+\frac{m_{k} \rho_{k}}{\rho_{k}^{2}\left(\cos \rho_{k}-\bar{\mu}\right)}\right)
$$

at $x=0, x=1$. Since $\sin \rho_{k}=\bar{\mu} \rho_{k}$ these series are

$$
\begin{aligned}
& \frac{2 A \bar{\mu}}{1-A^{2}} \sum_{k=K}^{k=\infty}\left(\frac{\alpha \cos \rho_{k}+\beta}{\cos \rho_{k}-\bar{\mu}}+\frac{m_{k} \rho_{k}}{\rho_{k}^{2}\left(\cos \rho_{k}-\bar{\mu}\right)}\right), \\
& \frac{2 \bar{\mu}}{1-A^{2}} \sum_{k=K}^{k=\infty}\left(\frac{\alpha \cos \rho_{k}+\beta}{\cos \rho_{k}-\bar{\mu}}+\frac{m_{k} \rho_{k}}{\rho_{k}^{2}\left(\cos \rho_{k}-\bar{\mu}\right)}\right) .
\end{aligned}
$$

Since $\cos \rho_{k}= \pm \sqrt{1-\sin ^{2} \rho_{k}}= \pm \sqrt{1-\bar{\mu}^{2} \rho_{k}^{2}}$ we have

$$
\liminf _{k \rightarrow \infty}\left|\frac{\cos \rho_{k}}{\cos \rho_{k}-\bar{\mu}}\right|>0,
$$

and the series $\sum \cos \rho_{k} /\left(\cos \rho_{k}-\bar{\mu}\right)$ is divergent. Similarly, we find

$$
\left|\frac{m_{k} \rho_{k}}{\rho_{k}{ }^{2}\left(\cos \rho_{k}-\bar{\mu}\right)}\right| \leqq \frac{M}{\left|\rho_{k}\right|^{2}} \quad(k=K, K+1, \cdots) .
$$

Since by Theorem IV $\sum 1 /\left|\rho_{k}\right|^{2}$ is comparable to $\sum 1 / k^{2}$, the series $\sum m_{k} \rho_{k} / \rho_{\mathbf{k}}^{2}\left(\cos \rho_{k}-\bar{\mu}\right)$ is convergent. Lastly we show that $\sum 1 /\left(\cos \rho_{k}-\bar{\mu}\right)$ converges. If $C_{l}$ is a simple closed contour on $\Sigma^{\prime}$ surrounding $\rho_{K}, \cdots, \rho_{K}+l$, then

$$
\sum_{k=K}^{k=K} \frac{1}{\cos \rho_{k}-\bar{\mu}}=\frac{1}{2 \pi i} \int_{C_{l}} \frac{d \rho}{\sin \rho-\bar{\mu} \rho}
$$

by the theory of residues. We shall take $C_{l}$ as being the contour made up of two concentric semi-circles on $\Sigma^{\prime}$, nameiy $\Gamma_{0}$ and $\gamma_{\mathrm{I}}+\gamma_{\mathrm{IV}}$, joined by segments of the imaginary axis which we shall call $\gamma_{1}$ and $\gamma_{4}$ respectively. We find

$$
\int_{\gamma_{1}} \frac{d \rho}{\sin \rho-\bar{\mu} \rho}=-\int_{\gamma_{1}} \frac{d \rho}{\sin \rho-\bar{\mu} \rho}
$$


by replacing $\rho$ by $-\rho$ in either integral. By work like that of Theorem XII we have

$$
\int_{\gamma_{\mathrm{I}}} \frac{d \rho}{\sin \rho-\bar{\mu} \rho}=\int_{\gamma_{\mathrm{I}}} \frac{2 i e^{\rho i} d \rho}{e^{2 \rho i}+\bar{\mu} \rho e^{\rho i}-1} \rightarrow 0, \int_{\gamma_{\mathrm{IV}}} \frac{d \rho}{\sin \rho-\bar{\mu} \rho} \rightarrow 0,
$$

as $R \rightarrow \infty$. By the use of these facts we find

$$
\lim _{l \rightarrow \infty} \sum_{k=K}^{k=K+l} \frac{1}{\cos \rho_{k}-\bar{\mu}}=\frac{1}{2 \pi i} \int_{\Gamma_{0}} \frac{d \rho}{\sin \rho-\bar{\mu} \rho},
$$

and the desired result is proved.

In short, if $\alpha=f(0)-A f(1)$ is different from zero, the expansions for $f(x)$, continuous together with its first three derivatives on $(0,1)$, in Case III diverge at $x=0$ and $x=1$, except when $A=0, x=0$; if $\alpha=0$ they converge, In Case III, therefore, the expansions present special irregularities at the end points of the interval of definition.

Columbia University,

NEW YORK, N.Y. 